\title{
Arabidopsis thaliana phytaspase: identification and peculiar properties
}

\begin{abstract}
Phytaspases are plant cell death-related proteases of the subtilisin-like protease family that possess an unusual aspartate cleavage specificity. Although phytaspase activity is widespread in plants, phytaspase of Arabidopsis thaliana (L.) Heynh. has escaped detection and identification thus far. Here, we show that a single gene (At4 g10540) out of 56 A. thaliana subtilisin-like protease genes encodes a phytaspase. The recombinant phytaspase was overproduced in Nicotiana benthamiana Domin leaves, isolated, and its substrate specificity and properties were characterised. At $\mathrm{pH}$ 5.5, at physiological mildly acidic reaction conditions, the Arabidopsis phytaspase was shown to be strictly Asp-specific. The strongly preferred cleavage motifs of the enzyme out of a panel of synthetic peptide substrates were YVAD and IETD, while the VEID-based substrate preferred by the tobacco and rice phytaspases was almost completely resistant to hydrolysis. At neutral $\mathrm{pH}$, however, the Arabidopsis phytaspase could hydrolyse peptide substrates after two additional amino acid residues, His and Phe, in addition to Asp. This observation may indicate that the repertoire of Arabidopsis phytaspase targets could possibly be regulated by the conditions of the cellular environment. Similar to tobacco and rice phytaspases, the Arabidopsis enzyme was shown to accumulate in the apoplast of epidermal leaf cells. However, in stomatal cells Arabidopsis phytaspase was observed inside the cells, possibly co-localising with vacuole. Our study thus demonstrates that the Arabidopsis phytaspase possesses both important similarities with and distinctions from the already known phytaspases, and is likely to be the most divergent member of the phytaspase family.
\end{abstract}

Keyword: Apoplast; Aspartate specificity; Proteolysis; Subtilisin-like protease 